\title{
Effects of Drying on the Biochemical Composition of Atherina boyeri from the Tunisian Coast
}

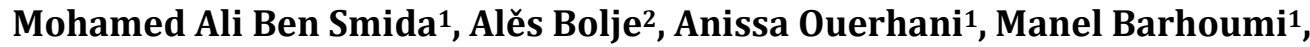 \\ Hassen Mejri', M'hamed El Cafsi' ${ }^{1}$, Rafika Fehri-Bedoui ${ }^{1}$ \\ ${ }^{1}$ Faculté des Sciences de Tunis, Université de Tunis El Manar, Tunis, Tunisie \\ ${ }^{2}$ Fisheries Research Institute of Slovenia, Ljubljana, Slovenia \\ Email: ali.bensmida@Laposte.net
}

Received 28 May 2014; revised 1 July 2014; accepted 13 July 2014

Copyright (C) 2014 by authors and Scientific Research Publishing Inc.

This work is licensed under the Creative Commons Attribution International License (CC BY). http://creativecommons.org/licenses/by/4.0/

(c) () Open Access

\begin{abstract}
The effects of solar and experimental drying on the protein concentration and total fatty acid (TFA) content of the muscles of Atherina boyeri (sand smelt) were studied. The percentage of protein was $18.3 \%$ for fresh fish and $14.2 \%$ for sun-dried fish, while TFA content was $4.9 \mathrm{~g} / 100 \mathrm{~g}$ and 0.5 $\mathrm{g} / \mathbf{1 0 0 g}$. After the drying experiment, the percentage of protein was $23 \%$ and the fatty acid (FA) content was $2.8 \mathrm{~g} / \mathbf{1 0 0 g}$. The results show that natural or experimental drying favors saturated fatty acids. The n-3 polyunsaturated fatty acids (PUFAs) are less sensitive to heat than the n-6 PUFAs. From a nutritional point of view, it seems that the drying conditions, where parameters are $T$ $=50^{\circ} \mathrm{C}$, moisture $=30 \%$ and air speed $=2 \mathrm{~m} / \mathrm{s}$, would be the most beneficial for the preservation of sand smelt.
\end{abstract}

\section{Keywords}

Biochemical Composition, Solar Drying, Drying Experimental, Atherina boyeri

\section{Introduction}

Fish is important for humans for its nutritional qualities and also for the choice it offers in taste [1], texture, and the form in which it is marketed: whole, fillets, fresh, frozen, salted, smoked, dried, or processed [2]. Fish meat is the part that is most used by consumers. The fish meat is the most used in part for human consumption. The lipids of the flesh have a beneficial effect on human health due to the presence of n-3 polyunsaturated fatty acids (PUFAs) [3].

How to cite this paper: Ben Smida, M.A., Bolje, A., Ouerhani, A., Barhoumi, M., Mejri, H., El Cafsi, M. and Fehri-Bedoui, R. (2014) Effects of Drying on the Biochemical Composition of Atherina boyeri from the Tunisian Coast. Food and Nutrition Sciences, 5, 1399-1407. http://dx.doi.org/10.4236/fns.2014.514152 
The nutritional qualities of fish are generally greater than or equal to those of meat, and the protein content of fish flesh is, regardless of the species, equivalent to that of meat [4]. In addition, fish protein is more digestible than meat protein and the levels of essential amino acids are generally a little higher than those of meat [5].

Proteins are able to soak up water and hold it against the force of gravity in a protein matrix [6]. Some species of fish have the ability to accumulate large amounts of fat to be used for the purposes of basic metabolism during migration and reproduction. The lipid content of a marketed species is essential information for consumers [7].

Fish is a perishable product, and its consumption is spread throughout the year [8]. To keep the fish for eating long after it was caught, it is necessary to preserve or transform the fish [9]. This process also requires drying, which aims to reduce the water content of the fish to promote its preservation.

Water is a vector of bacterial infections, and chemical, and biochemical decomposition, and is involved in the degradation reactions of the product. It is therefore necessary to partially dehydrate the product to stabilize, by removing a portion of the so-called "free" water. Proper drying can only be achieved all year round only if the temperature, humidity, and ventilation are controlled. Without control of these three parameters, the drying time can be very long, depending on the climatic conditions [10].

In Tunisia, sand smelt is known as the "Cherkaou" and is consumed in the areas of Monastir, Djerba, and Gabes. It is preserved by conventional drying and is consumed in this form [11]. Given the importance of fatty acids and proteins for the proper functioning of the human body, it is of interest to quantify the fatty acid and protein contents of the flesh of $A$. boyeri. In this study, we compared the effects of traditional and artificial drying on the protein and fatty acid contents of $A$. boyeri.

\section{Materials and Methods}

\subsection{Sampling and Biological Parameters}

Fresh samples of $A$. boyeri came from the region of Monastir. These samples were collected in winter 2010. The average total length (TL) and the average total weights (TW) of the individuals are shown in Table 1.

\subsection{Drying Conditions}

The fish were divided into several groups, each consisting of six samples $(n=6)$. One group was subjected to solar drying (SN). Another group was analyzed fresh (F). The other fish was subjected to different experimental conditions. It was dehydrated by convective drying at variable air speeds $(1 \mathrm{~m} / \mathrm{s}$ and $2 \mathrm{~m} / \mathrm{s})$ at different temperatures $\left(45^{\circ} \mathrm{C}, 50^{\circ} \mathrm{C}, 60^{\circ} \mathrm{C}\right.$, and $\left.70^{\circ} \mathrm{C}\right)$ and relative humidity $(20 \%, 30 \%$, and $40 \%)$. The experimental conditions are summarized in Table 2 .

The drying tests were conducted in a closed-loop drying system using forced hot air convection. The average diameter of the fish was $14 \mathrm{~mm}$, and the initial water content varied between $75 \%$ and $80 \%$. The conditions of the drying air were kept constant during each test. A high precision balance is equipped with a data output enables via a software acquisition weight during the drying process. The temperature inside and on the surface of $A$. boyeri was measured using thermocouples connected to a data acquisition channel. The air flow in the test section was perpendicular to the surface of the product to be dried in order to obtain optimum air-fish contact.

\subsection{Determination of Moisture Content}

This measurement was made on fresh fish and dried fish. The fish was weighed prior to being placed in an oven to determine the wet weight $\left(P_{h}\right)$. It was then placed in an oven at a temperature of $105^{\circ} \mathrm{C}$ for 24 hours until a constant weight was reached $\left(P_{s}\right)$. Moisture was calculated using the following formula:

$$
\text { Moisture }(\%)=\left[\frac{P_{h}-P_{s}}{P_{h}}\right] \times 100
$$

\subsection{Extraction of Total Fatty Acids}

The extraction of total fatty acids (TFAs) was performed in the presence of chloroform-methanol (2:1 v/v) [12]. The total lipids obtained were stored in chloroform-methanol-butylatedhydroxytoluene (BHT) at $-28^{\circ} \mathrm{C}$. For 
Table 1. Biometric features A. boyeri.

\begin{tabular}{ccc}
\hline & TL $(\mathrm{mm})$ & TW $(\mathrm{g})$ \\
\hline Winter 2010 & $40-65$ & $0.45-5.73 \mathrm{~g}$ \\
\hline
\end{tabular}

Table 2. Experimental drying conditions.

\begin{tabular}{|c|c|c|c|c|c|c|c|c|c|}
\hline \multicolumn{10}{|c|}{ Drying conditions } \\
\hline Parameters & E1 & E2 & E6 & E8 & E9 & E4 & E3 & E5 & E7 \\
\hline Temperature ${ }^{\circ} \mathrm{C}$ & 70 & 60 & 50 & 45 & 60 & 70 & 60 & 50 & 45 \\
\hline Moisture \% & 20 & 20 & 20 & 20 & 20 & 30 & 30 & 30 & 30 \\
\hline Air speed m/s & 2 & 2 & 2 & 2 & 1 & 2 & 2 & 2 & 2 \\
\hline
\end{tabular}

further analysis, the fatty acids were transformed into methyl esters, according to Cecchi et al. [13]. The quantification of the fatty acids is based on an internal standard not present in our samples, methyl nonadecanoate or $\mathrm{C}_{19: 0}$ (Sigma Aldrich, Corporate Headquarters, St. Louis, MO).

\subsection{Identification and Quantification of Fatty Acids}

Methyl esters of TFAs were separated, identified, and quantitated by gas chromatography using a HP 6890 gas chromatograph with a split/splitless injector with electronic pressure control and a flame ionisation detector was used for the analysis. Separation was performed with a $30 \mathrm{~m}$ HP Innowax capillary column with an internal diameter of $250 \mu \mathrm{m}$ and a $0.25 \mu \mathrm{m}$ film thickness, the stationary polar phase of the column being polyethylene glycol.

\subsection{Protein Determination}

The determination of protein content was based on a solution of serum albumin [14]. The sample was incubated for 30 minutes away from light and then the optical density at a $540 \mathrm{~nm}$ was measured.

\subsection{Statistical Analysis}

Mean comparison was performed by analysis of variance (ANOVA) followed by Duncan test at the significance level of $\mathrm{p}<0.05$. All statistical analyses were carried out using the software program SPSS version 13 (SPSS Inc., Chicago, Illinois).

\section{Results}

The percentages of moisture protein and total fatty acids (TFA) of fresh and sun-dried A. boyeri are shown in Table 3.

\subsection{Effects of Experimental Drying on Protein Content}

After subjecting A. boyeri to different experimental drying conditions, the protein content of the flesh varied significantly. The results are shown in Table 4.

A maximum of $23 \%$ protein was obtained with E2. The effects of different temperatures were observed with E2 (23\%), E6 (18\%), and E8 (18\%); the difference between the three conditions was significant. Protein content varied significantly with moisture, with a significant difference between E2 (23\%) and E3 (19.6\%). The drying rate appeared to have a destructive effect on the protein content; the destructive effect of protein content was greater with low drying speed of $1 \mathrm{~m} / \mathrm{s}$. the percentages were 23\% and 17.6\% for E2 and E9, respectively.

\subsection{Effects of Drying on TFA Content}

TFA content after extraction was expressed in g per $100 \mathrm{~g}$ of fish. The TFA contents in fresh and sun-dried $A$. boyeri were $4.9 \pm 1.9 \mathrm{~g} / 100 \mathrm{~g}$ and $0.5 \pm 0.0 \mathrm{~g} / 100 \mathrm{~g}$ respectively (Table 3). Drying may be a necessary step in food preservation. We subjected $A$. boyeri to different experimental drying conditions. The TFAs obtained are shown in Table 4. Drying appeared to affect the TFA content of the flesh of $A$. boyeri. The values varied from $4.9 \mathrm{~g} / 100 \mathrm{~g}$ 
Table 3. Water (\%), protein (\%), and total fatty acid content (g/100g) of fresh and sun-dried A. boyeri (mean \pm SE, $n=6)$.

\begin{tabular}{cccc}
\hline & \multicolumn{3}{c}{ Component } \\
\hline & Moisture & Protein & TFA \\
\hline A. boyeri fresh & $77.3 \pm 0.3$ & $18.3 \pm 1.4$ & $4.9 \pm 1.9$ \\
A. boyeri sun-dried & $16.4 \pm 0.5$ & $14.2 \pm 0.3$ & $0.5 \pm 0.0$ \\
\hline
\end{tabular}

Table 4. Comparative table of protein content (\%) and total fatty acid content (g/100g) of fish dried at different experimental conditions (mean $\pm S E, n=6$; superscript letters indicate inter group statistical differences, $p<0.05$ ).

\begin{tabular}{cccccccccc}
\hline \multicolumn{8}{c}{ Drying conditions } \\
\hline & E1 & E2 & E3 & E4 & E5 & E6 & E7 & E8 & E9 \\
\hline Protein & $22.5 \pm 4.2^{\mathrm{ab}}$ & $23 \pm 0.7^{\mathrm{a}}$ & $19.6 \pm 1^{\mathrm{abc}}$ & $19.5 \pm 2.5^{\mathrm{abc}}$ & $15.6 \pm 1^{\mathrm{c}}$ & $18.3 \pm 0.8^{\mathrm{abc}}$ & $14.8 \pm 0.6^{\mathrm{c}}$ & $18.2 \pm 0.7^{\mathrm{abc}}$ & $17.6 \pm 0.9^{\mathrm{bc}}$ \\
TFA & $1.8 \pm 0.4^{\mathrm{ab}}$ & $2.8 \pm 0.5^{\mathrm{a}}$ & $2.6 \pm 0.5^{\mathrm{a}}$ & $2.6 \pm 0.5^{\mathrm{a}}$ & $1.8 \pm 0.3^{\mathrm{ab}}$ & $1.1 \pm 0.2^{\mathrm{b}}$ & $1.4 \pm 0.3^{\mathrm{ab}}$ & $1.1 \pm 0.2^{\mathrm{b}}$ & $2.6 \pm 0.3^{\mathrm{a}}$ \\
\hline
\end{tabular}

in fresh fish, depending on the experimental conditions. Conditions E2, E3, E4 and E9 seem to be the best for the preservation of TFAs in A. boyeri; TFA content varied from 2.6 to $2.8 \mathrm{~g} / 100 \mathrm{~g}$.

Variations in temperature and moisture content led to significant variations in TFA content between the experimental conditions. At $60^{\circ} \mathrm{C}$, the moisture content did not cause significant variation between E2 $(2.8 \mathrm{~g} / 100 \mathrm{~g})$ and E3 $(2.6 \mathrm{~g} / 100 \mathrm{~g})$. By contrast, at $70^{\circ} \mathrm{C}$, a significant difference was found between $\mathrm{E} 4$ and $\mathrm{E} 1$ where the TFA content was $2.6 \mathrm{~g} / 100 \mathrm{~g}$ and $1.8 \mathrm{~g} / 100 \mathrm{~g}$, respectively. The drying rate did not seem to affect the TFA content. No significant difference was found between E3 and E9.

\subsection{A. boyeri Lipid Profile}

Under experimental drying condition (E1 - E9), 22 fatty acids were identified in the tissues of A. boyeri (Table 5). Five fatty acids were particularly prominent in the lipid composition of fresh fish. The palmitic fatty acid $\left(\mathrm{C}_{16: 0}\right)$ $(20.2 \%-26.9 \%)$ and stearic acid $\left(\mathrm{C}_{18: 0}\right)(7.8 \%-10.4 \%)$ predominated among the saturated fatty acids (SFAs). Oleic acid $\left(\mathrm{C}_{18: 1 n-9}\right)$ was the main component of the monounsaturated fatty acids (MUFAs), varying between $8.9 \%$ and $14.4 \%$ of the TFAs. It was followed by vaccenic acid $\left(\mathrm{C}_{18: 1 \mathrm{n}-7}\right)$ and palmitoleic acid $\left(\mathrm{C}_{16: 1 \mathrm{n}-7}\right)$. Among the PUFAs, the majority were docosahexaenoic fatty acids (DHA, $\left.\mathrm{C}_{22: 6 n-3}\right)$ and eicosapentaenoic acid EPA $\left(\mathrm{C}_{20: 5 n-3}\right)$. Their cumulative percentages varied between $6.8 \%$ and $25.5 \%$ of the TFAs. Natural drying (SN) promoted the formation of saturated fatty acids (SFA) with palmitic acid $\mathrm{C}_{16: 0}$ as the predominant fatty acid; oleic acid was the predominant MUFA; and DHA was the predominant PUFA.

\subsection{Composition of Fatty Acid Groups}

\subsubsection{In Fresh Fish and Sun-Dried Fish}

Natural drying (SN) promotes the formation of saturated fatty acids that reach a percentage of $67.2 \%$, while in the fresh fish, the percentage of saturated fatty acids is $42.6 \%$ of the TFAs (Table 5).

\subsubsection{Under Experimental Drying}

For fresh fish, natural drying seemed to affect MUFA content; while PUFA content remained stable (Figure 1). There seemed to be an inverse relationship between the percentage of SFAs and MUFAs. The most favorable conditions for the preservation of $A$. boyeri were those that showed a minimum percentage of SFA and a maximum percentage of unsaturated fatty acids (MUFA + PUFA). The results of E5, E6, and E7 met these conditions.

According to Figure 2, solar drying (SN) affected the percentage of unsaturated fatty acids. The PUFAs that were most sensitive to experimental drying were those of the n- 6 family. Fatty acids of the n-3 family did not seem to be affected by experimental drying; the content was higher than in fresh fish. Two points stood out, E1 and E5, with respective percentages of 30.1 and $30.4 \%$ of the TFAs. Among the MUFAs, n-9 fatty acids were more resistant to experimental drying than $\mathrm{n}-7$ fatty acids.

We can also see from Table 5 that the n-3/n-6 ratio varied between 2.9 and 4.8. The highest ratios of 4.2 and 4.8 were observed in E5 and E8, respectively, which had the lowest percentages of n-6 fatty acids (7.5\% and 6.8\% of the TFAs, respectively). Of interest from a nutritional point of view, the levels of EPA + DHA were expressed 


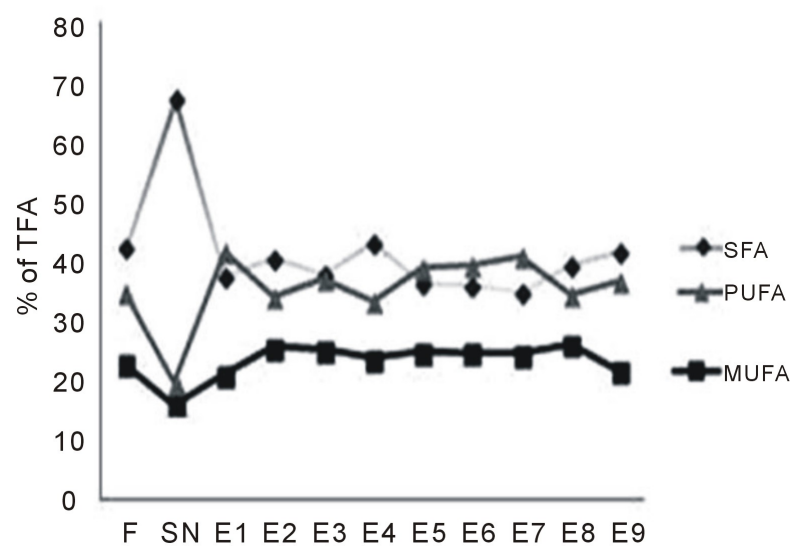

Figure 1. Percentages of saturated fatty acids (SFA), monounsaturated fatty acids (MUFA) and polyunsaturated fatty acids (PUFA) under different experimental conditions.

Table 5. Fatty acid profile under different experimental conditions (\%TFA, values with superscript letters indicate inter group statistical differences, $\mathrm{p}<0.05$ ).

\begin{tabular}{|c|c|c|c|c|c|c|}
\hline \multicolumn{7}{|c|}{ Drying condition } \\
\hline Fatty acid & $\mathrm{F}$ & $\mathrm{SN}$ & E1 & E2 & E3 & E4 \\
\hline $\mathrm{C}_{14: 0}$ & $3.8 \pm 0.8^{\mathrm{a}}$ & $2.0 \pm 1.1^{\mathrm{a}}$ & $2.7 \pm 0.4^{\mathrm{a}}$ & $3.7 \pm 0.6^{\mathrm{a}}$ & $3.5 \pm 0.5^{\mathrm{a}}$ & $4.2 \pm 0.5^{\mathrm{a}}$ \\
\hline $\mathrm{C}_{15: 0}$ & $1.0 \pm 0.2^{\mathrm{a}}$ & $1.2 \pm 0.1^{\mathrm{a}}$ & $0.9 \pm 0.1^{\mathrm{a}}$ & $0.9 \pm 0.2^{\mathrm{a}}$ & $1.0 \pm 0.1^{\mathrm{a}}$ & $1.1 \pm 0.1^{\mathrm{a}}$ \\
\hline $\mathrm{C}_{16: 0}$ & $24.5 \pm 3.5^{\mathrm{ab}}$ & $34.6 \pm 2.5^{\mathrm{a}}$ & $23.9 \pm 0.9^{\mathrm{ab}}$ & $26.1 \pm 0.8^{\mathrm{ab}}$ & $23.8 \pm 0.6^{\mathrm{ab}}$ & $26.9 \pm 1.6^{\mathrm{ab}}$ \\
\hline $\mathrm{C}_{16: 1 \mathrm{n}-7}$ & $5.6 \pm 1.6^{\mathrm{a}}$ & $2.1 \pm 1.0^{\mathrm{b}}$ & $5.0 \pm 0.5^{\mathrm{a}}$ & $6.7 \pm 0.4^{\mathrm{a}}$ & $6.8 \pm 1.0^{\mathrm{a}}$ & $6.8 \pm 0.7^{\mathrm{a}}$ \\
\hline$C_{16: 2 n-4}$ & $0.7 \pm 0.2^{b}$ & $4.0 \pm 1.7^{\mathrm{a}}$ & $0.7 \pm 0.2^{b}$ & $0.8 \pm 0.2^{b}$ & $0.7 \pm 0.2^{\mathrm{b}}$ & $0.7 \pm 0.2^{b}$ \\
\hline $\mathrm{C}_{17: 0}$ & $0.9 \pm 0.2$ & $1.0 \pm 0.2$ & $0.6 \pm 0.1$ & $1.0 \pm 0.2$ & $0.6 \pm 0.1$ & $0.6 \pm 0.1$ \\
\hline$C_{16: 3 n-4}$ & $0.4 \pm 0.2^{\mathrm{ab}}$ & $0.8 \pm 0.4^{\mathrm{a}}$ & $0.2 \pm 0.0^{\mathrm{ab}}$ & $0.3 \pm 0.1^{\mathrm{ab}}$ & $0.1 \pm 0.0^{\mathrm{b}}$ & $0.1 \pm 0.0^{\mathrm{b}}$ \\
\hline$C_{16: 4 n-3}$ & $0.4 \pm 0.1^{\mathrm{a}}$ & $2.8 \pm 1.0^{\mathrm{a}}$ & $0.2 \pm 0.0^{\mathrm{a}}$ & $0.3 \pm 0.1^{\mathrm{a}}$ & $0.3 \pm 0.0^{\mathrm{a}}$ & $0.4 \pm 0.1^{\mathrm{a}}$ \\
\hline $\mathrm{C}_{18: 0}$ & $12.5 \pm 3.5^{b}$ & $28.4 \pm 5.0^{\mathrm{a}}$ & $9.5 \pm 0.5^{b}$ & $8.7 \pm 0.4^{b}$ & $8.8 \pm 0.5^{b}$ & $10.4 \pm 1.4^{\mathrm{b}}$ \\
\hline $\mathrm{C}_{18: 1 \mathrm{n}-9}$ & $10.0 \pm 2.1^{\mathrm{ab}}$ & $8.9 \pm 2.1^{b}$ & $11.6 \pm 0.4^{\mathrm{ab}}$ & $12.9 \pm 1.0^{\mathrm{ab}}$ & $12.6 \pm 0.5^{\mathrm{ab}}$ & $11.6 \pm 0.9^{\mathrm{ab}}$ \\
\hline $\mathrm{C}_{18: 1 \mathrm{n}-7}$ & $6.4 \pm 2.2^{\mathrm{a}}$ & $5.0 \pm 0.9^{\mathrm{a}}$ & $4.1 \pm 0.2^{\mathrm{a}}$ & $5.4 \pm 0.4^{\mathrm{a}}$ & $5.3 \pm 0.5^{\mathrm{a}}$ & $4.8 \pm 0.4^{\mathrm{a}}$ \\
\hline$C_{18: 2 n-6}$ & $5.1 \pm 2.3^{\mathrm{a}}$ & $1.5 \pm 0.4^{\mathrm{b}}$ & $2.4 \pm 0.2^{\mathrm{b}}$ & $2.2 \pm 0.1^{b}$ & $2.9 \pm 0.4^{\mathrm{ab}}$ & $3.0 \pm 0.5^{\mathrm{ab}}$ \\
\hline $\mathrm{C}_{18: 3 n-3}$ & $1.7 \pm 0.4^{\mathrm{a}}$ & $0.5 \pm 021^{\mathrm{c}}$ & $1.2 \pm 0.1^{\mathrm{abc}}$ & $1.2 \pm 0.1^{\mathrm{abc}}$ & $1.3 \pm 0.2^{\mathrm{ab}}$ & $1.3 \pm 0.1^{\mathrm{ab}}$ \\
\hline $\mathrm{C}_{18: 4 \mathrm{n}-3}$ & $1.4 \pm 0.2^{\mathrm{ab}}$ & $0.2 \pm 0.1^{\mathrm{c}}$ & $1.1 \pm 0.2^{\mathrm{ab}}$ & $1.0 \pm 0.1^{\mathrm{ab}}$ & $1.0 \pm 0.1^{\mathrm{ab}}$ & $1.3 \pm 0.3^{\mathrm{ab}}$ \\
\hline $\mathrm{C}_{20: 1 \mathrm{n}-9}$ & $0.6 \pm 0.3^{\mathrm{ab}}$ & trace & $0.4 \pm 0.1^{\mathrm{abc}}$ & $0.6 \pm 0.1^{\mathrm{ab}}$ & $0.5 \pm 0.1^{\mathrm{ab}}$ & $0.4 \pm 0.1^{\mathrm{abc}}$ \\
\hline $\mathrm{C}_{20: 2 \mathrm{n}-6}$ & $0.4 \pm 0.1^{\mathrm{bc}}$ & trace & $0.5 \pm 0.0^{\mathrm{bc}}$ & $0.5 \pm 0 .^{\mathrm{bc}}$ & $0.5 \pm 0.1^{\mathrm{bc}}$ & $0.3 \pm 0.1^{\mathrm{bc}}$ \\
\hline$C_{20: 4 n-6}$ & $5.0 \pm 1.3^{\mathrm{ab}}$ & $2.7 \pm 0.7^{\mathrm{C}}$ & $6.0 \pm 0.7^{\mathrm{ab}}$ & $4.7 \pm 0.6^{\mathrm{abc}}$ & $5.4 \pm 0.4^{\mathrm{ab}}$ & $4.5 \pm 0.6^{\mathrm{abc}}$ \\
\hline$C_{20: 4 n-3}$ & $0.4 \pm 0.1^{\mathrm{ab}}$ & trace & $0.5 \pm 0.0^{\mathrm{ab}}$ & $0.5 \pm 0.1^{\mathrm{a}}$ & $0.5 \pm 0.0^{\mathrm{a}}$ & $0.4 \pm 0.0^{\mathrm{ab}}$ \\
\hline $\mathrm{C}_{20: 5 n-3}$ & $6.3 \pm 0.7^{\mathrm{a}}$ & $1.7 \pm 0.6^{b}$ & $7.0 \pm 0.3^{\mathrm{a}}$ & $6.2 \pm 0.7^{\mathrm{a}}$ & $5.9 \pm 0.3^{\mathrm{a}}$ & $5.7 \pm 0.3^{\mathrm{a}}$ \\
\hline $\mathrm{C}_{22: 5 \mathrm{n}-6}$ & $0.9 \pm 0.3^{b}$ & trace & $1.2 \pm 0.2^{\mathrm{b}}$ & $1.1 \pm 0.3^{\mathrm{b}}$ & $1.1 \pm 0.1^{\mathrm{b}}$ & $0.8 \pm 0.1^{\mathrm{b}}$ \\
\hline $\mathrm{C}_{22: 5 n-3}$ & $1.4 \pm 0.3^{\mathrm{a}}$ & trace & $1.9 \pm 0.1^{\mathrm{a}}$ & $1.7 \pm 0.3^{\mathrm{a}}$ & $2.0 \pm 0.1^{\mathrm{a}}$ & $1.4 \pm 0.2^{\mathrm{a}}$ \\
\hline $\mathrm{C}_{22: 6 n-3}$ & $10.6 \pm 0.3^{c}$ & $5.2 \pm 1.5^{c}$ & $18.4 \pm 1.1^{\mathrm{abc}}$ & $13.5 \pm 1.2^{\mathrm{bc}}$ & $15.4 \pm 1.7^{\mathrm{abc}}$ & $13.2 \pm 1.2^{\mathrm{bc}}$ \\
\hline SFA & $42.6 \pm 6.1^{\mathrm{b}}$ & $67.2 \pm 4.4^{\mathrm{a}}$ & $37.6 \pm 1.4^{\mathrm{b}}$ & $40.4 \pm 2.9^{\mathrm{b}}$ & $37.7 \pm 1.0^{\mathrm{b}}$ & $43.2 \pm 2.9^{b}$ \\
\hline MUFA & $22.7 \pm 2.7^{\mathrm{a}}$ & $16.0 \pm 2.1^{\mathrm{b}}$ & $21.0 \pm 0.8^{\mathrm{ab}}$ & $25.6 \pm 1.5^{\mathrm{a}}$ & $25.2 \pm 1.6^{\mathrm{a}}$ & $23.6 \pm 1.8^{\mathrm{a}}$ \\
\hline n-9 & $10.6 \pm 2^{\mathrm{ab}}$ & $8.9 \pm .2 .1^{\mathrm{b}}$ & $12.0 \pm 0.4^{\mathrm{ab}}$ & $13.5 \pm 1.0^{\mathrm{ab}}$ & $13.1 \pm 0.5^{\mathrm{a}}$ & $12.0 \pm 0.9^{\mathrm{ab}}$ \\
\hline$n-7$ & $12.1 \pm 1.5^{\mathrm{a}}$ & $7.1 \pm 0.9^{\mathrm{b}}$ & $9.1 \pm 0.6^{\mathrm{ab}}$ & $12.1 \pm 0.6^{\mathrm{a}}$ & $12.1 \pm 1.5^{\mathrm{a}}$ & $11.6 \pm 0.1^{\mathrm{a}}$ \\
\hline PUFA & $34.7 \pm 4.4^{\mathrm{a}}$ & $19.5 \pm 4.1^{\mathrm{b}}$ & $41.4 \pm 1.9^{\mathrm{a}}$ & $34.0 \pm 2.7^{\mathrm{ab}}$ & $37.1 \pm 2.3^{\mathrm{a}}$ & $33.2 \pm 1.8^{\mathrm{a}}$ \\
\hline$n-3$ & $22.1 \pm 2.6^{\mathrm{a}}$ & $10.3 \pm 3.5^{\mathrm{b}}$ & $30.1 \pm 1.4^{\mathrm{a}}$ & $24.4 \pm 1.8^{\mathrm{a}}$ & $26.3 \pm 1.8^{\mathrm{a}}$ & $23.7 \pm 1.7^{\mathrm{a}}$ \\
\hline$n-6$ & $11.4 \pm 1.9^{\mathrm{a}}$ & $4.3 \pm 0.8^{c}$ & $10.1 \pm 0.9^{\mathrm{ab}}$ & $8.5 \pm 0.9^{\mathrm{ab}}$ & $9.9 \pm 0.8^{\mathrm{ab}}$ & $8.6 \pm 0.9^{\mathrm{ab}}$ \\
\hline$n-3 / n-6$ & $2.1^{\mathrm{b}}$ & $3.0^{\mathrm{ab}}$ & $3.1^{\mathrm{ab}}$ & $3^{\mathrm{ab}}$ & $2.9^{\mathrm{ab}}$ & $2.9^{\mathrm{ab}}$ \\
\hline $\mathrm{EPA}+\mathrm{DHA}$ & $16.9 \pm 2.3^{\mathrm{a}}$ & $6.8 \pm 1.8^{\mathrm{b}}$ & $25.5 \pm 1.4^{\mathrm{a}}$ & $19.8 \pm 1.5^{\mathrm{a}}$ & $21.3 \pm 1.9^{\mathrm{a}}$ & $18.9 \pm 1.4^{\mathrm{a}}$ \\
\hline $\mathrm{EPA}+\mathrm{DHA}^{*}$ & 680 & 27 & 459 & 554 & 553 & 491 \\
\hline PI & 176 & 83 & 246 & 195 & 213 & 187 \\
\hline $\mathrm{HH}$ & 1.43 & 0.56 & 1.82 & 1.49 & 1.66 & 1.30 \\
\hline
\end{tabular}




\section{Continued}

\begin{tabular}{|c|c|c|c|c|c|}
\hline \multicolumn{6}{|c|}{ Drying condition } \\
\hline Fatty acid & E5 & E6 & E7 & E8 & E9 \\
\hline $\mathrm{C}_{14: 0}$ & $4.4 \pm 0.5^{\mathrm{a}}$ & $3.4 \pm 0.5^{\mathrm{a}}$ & $4.4 \pm 1.0^{\mathrm{a}}$ & $3.9 \pm 0.3^{\mathrm{a}}$ & $4.1 \pm 0.3^{\mathrm{a}}$ \\
\hline $\mathrm{C}_{15: 0}$ & $1.1 \pm 0.1^{\mathrm{a}}$ & $0.9 \pm 0.1^{\mathrm{a}}$ & $1.0 \pm 0.1^{\mathrm{a}}$ & $1.2 \pm 0.1^{\mathrm{a}}$ & $1.1 \pm 0.1^{\mathrm{a}}$ \\
\hline $\mathrm{C}_{16: 0}$ & $22.2 \pm 1.2^{\mathrm{ab}}$ & $21.4 \pm 1.4^{\mathrm{b}}$ & $20.2 \pm 1.6^{\mathrm{b}}$ & $23.3 \pm 0.9^{\mathrm{ab}}$ & $26.5 \pm 0.8^{\mathrm{ab}}$ \\
\hline $\mathrm{C}_{16: 1 \mathrm{n}-7}$ & $6.9 \pm 0.7^{\mathrm{a}}$ & $5.8 \pm 0.5^{\mathrm{a}}$ & $6.3 \pm 0.4^{\mathrm{a}}$ & $6.7 \pm 0.4^{\mathrm{a}}$ & $6.5 \pm 0.4^{\mathrm{a}}$ \\
\hline $\mathrm{C}_{16: 2 \mathrm{n}-4}$ & $0.7 \pm 0.1^{\mathrm{b}}$ & $0.4 \pm 0.0^{\mathrm{b}}$ & $0.5 \pm 0.1^{b}$ & $0.4 \pm 0.1^{\mathrm{b}}$ & $0.6 \pm 0.0^{\mathrm{b}}$ \\
\hline $\mathrm{C}_{17: 0}$ & $0.8 \pm 0.2$ & $0.4 . \pm 0.2$ & $0.6 \pm 0.2$ & $0.9 \pm 0.3$ & $0.8 \pm 0.0$ \\
\hline$C_{16: 3 n-4}$ & $0.3 \pm 0.1^{\mathrm{ab}}$ & $0.1 \pm 0.0^{\mathrm{b}}$ & $0.2 \pm 0.0^{\mathrm{ab}}$ & $0.3 \pm 0.2^{\mathrm{ab}}$ & $0.2 \pm 0.0^{\mathrm{b}}$ \\
\hline $\mathrm{C}_{16: 4 \mathrm{n}-3}$ & $0.4 \pm 0.1^{\mathrm{a}}$ & $0.2 \pm 0.0^{\mathrm{a}}$ & $0.8 \pm 0.4^{\mathrm{a}}$ & $0.3 \pm 0.1^{\mathrm{a}}$ & $0.2 \pm 0.1^{\mathrm{a}}$ \\
\hline $\mathrm{C}_{18: 0}$ & $7.8 \pm 0.3^{b}$ & $10.0 \pm 0.6^{\mathrm{b}}$ & $8.6 \pm 0.7^{b}$ & $10.3 \pm 1.5^{\mathrm{b}}$ & $9.3 \pm 0.8^{\mathrm{b}}$ \\
\hline $\mathrm{C}_{18: 1 \mathrm{n}-9}$ & $12.5 \pm 0.8^{\mathrm{ab}}$ & $14.2 \pm 0.2^{\mathrm{a}}$ & $13.2 \pm 0.7^{\mathrm{ab}}$ & $14.4 \pm 1.1^{\mathrm{a}}$ & $10.8 \pm 1.3^{\mathrm{ab}}$ \\
\hline $\mathrm{C}_{18: 1 \mathrm{n}-7}$ & $4.6 \pm 0.5^{\mathrm{a}}$ & $4.4 \pm 0.4^{\mathrm{a}}$ & $4.6 \pm 0.3^{\mathrm{a}}$ & $4.5 \pm 0.4^{\mathrm{a}}$ & $3.8 \pm 0.8^{\mathrm{a}}$ \\
\hline $\mathrm{C}_{18: 2 \mathrm{n}-6}$ & $2.6 \pm 0.3^{\mathrm{ab}}$ & $2.4 \pm 0.1^{\mathrm{b}}$ & $2.2 \pm 0.1^{\mathrm{b}}$ & $2.3 \pm 0.4^{\mathrm{b}}$ & $2.3 \pm 0.2^{\mathrm{b}}$ \\
\hline $\mathrm{C}_{18: 3 \mathrm{n}-3}$ & $1.5 \pm 0.2^{\mathrm{ab}}$ & $1.0 \pm 0.1^{\mathrm{bc}}$ & $1.0 \pm 0.0^{\mathrm{bc}}$ & $1.1 \pm 0.0^{\mathrm{abc}}$ & $1.2 \pm 0.1^{\mathrm{abc}}$ \\
\hline $\mathrm{C}_{18: 4 \mathrm{n}-3}$ & $1.4 \pm 0.3^{\mathrm{a}}$ & $0.9 \pm 0.1^{\mathrm{abc}}$ & $0.7 \pm 0.1^{\mathrm{bc}}$ & $0.8 \pm 0.2^{\mathrm{abc}}$ & $1.0 \pm 0.2^{\mathrm{ab}}$ \\
\hline $\mathrm{C}_{20: 1 \mathrm{n}-9}$ & $0.8 \pm 0.3^{\mathrm{a}}$ & $0.2 \pm 0.0^{\mathrm{bc}}$ & $0.4 \pm 0.1^{\mathrm{abc}}$ & $0.5 \pm 0.1^{\mathrm{ab}}$ & $0.4 \pm 0.0^{\mathrm{abc}}$ \\
\hline $\mathrm{C}_{20: 2 \mathrm{n}-6}$ & $1.0 \pm 0.4^{\mathrm{a}}$ & $0.4 \pm 0.0^{\mathrm{bc}}$ & $0.7 \pm 0.2^{\mathrm{b}}$ & $0.4 \pm 0.1^{\mathrm{bc}}$ & $0.4 \pm 0.0^{\mathrm{bc}}$ \\
\hline $\mathrm{C}_{20: 4 \mathrm{n}-6}$ & $2.9 \pm 0.6^{\mathrm{bc}}$ & $6.0 \pm 0.9^{\mathrm{a}}$ & $4.2 \pm 0.5^{\mathrm{abc}}$ & $3.2 \pm 0.2^{\mathrm{abc}}$ & $4.5 \pm 0.4^{\mathrm{abc}}$ \\
\hline $\mathrm{C}_{20: 4 \mathrm{n}-3}$ & $0.8 \pm 0.4^{\mathrm{a}}$ & $0.4 \pm 0.0^{\mathrm{ab}}$ & $0.5 \pm 0.1^{\mathrm{a}}$ & $0.5 \pm 0.0^{\mathrm{a}}$ & $0.5 \pm 0.0^{\mathrm{a}}$ \\
\hline $\mathrm{C}_{20: 5 n-3}$ & $5.0 \pm 0.3^{\mathrm{a}}$ & $5.8 \pm 0.3^{\mathrm{a}}$ & $5.1 \pm 0.3^{\mathrm{a}}$ & $4.6 \pm 0.2^{\mathrm{a}}$ & $5.8 \pm 0.1^{\mathrm{a}}$ \\
\hline $\mathrm{C}_{22: 5 n-6}$ & $1.0 \pm 0.1^{\mathrm{b}}$ & $1.2 \pm 0.2^{\mathrm{b}}$ & $4.4 \pm 3.1^{\mathrm{a}}$ & $0.8 \pm 0.1^{\mathrm{b}}$ & $1.1 \pm 0.1^{b}$ \\
\hline $\mathrm{C}_{22: 5 n-3}$ & $1.7 \pm 0.1^{\mathrm{a}}$ & $1.6 \pm 0.2^{\mathrm{a}}$ & $2.9 \pm 1.3^{\mathrm{a}}$ & $1.8 \pm 0.2^{\mathrm{a}}$ & $1.6 \pm 0.2^{\mathrm{a}}$ \\
\hline$C_{22: 6 n-3}$ & $19.7 \pm 2.6^{\mathrm{a}}$ & $18.8 \pm 1.1^{\mathrm{abc}}$ & $17.5 \pm 1.9^{\mathrm{abc}}$ & $17.7 \pm 2^{\mathrm{ab}}$ & $17.3 \pm 1.0^{\mathrm{abc}}$ \\
\hline SFA & $36.2 \pm 1.6^{\mathrm{b}}$ & $36.1 \pm 1.9^{b}$ & $34.8 \pm 2.8^{\mathrm{b}}$ & $39.5 \pm 2.3^{b}$ & $41.8 \pm 1.7^{\mathrm{b}}$ \\
\hline MUFA & $24.8 \pm 1.4^{\mathrm{a}}$ & $24.6 \pm 0.6^{\mathrm{a}}$ & $24.4 \pm 1.3^{\mathrm{a}}$ & $26.0 \pm 1.8^{\mathrm{a}}$ & $21.6 \pm 1.7^{\mathrm{ab}}$ \\
\hline n-9 & $13.3 \pm 0.6^{\mathrm{a}}$ & $14.4 \pm 0.2^{\mathrm{a}}$ & $13.5 \pm 0.7^{\mathrm{ab}}$ & $14.9 \pm 1.2^{\mathrm{a}}$ & $11.3 \pm 1.2^{\mathrm{ab}}$ \\
\hline$n-7$ & $11.5 \pm 1.0^{\mathrm{a}}$ & $10.2 \pm 0.7^{\mathrm{ab}}$ & $10.9 \pm 0.7^{\mathrm{a}}$ & $11.2 \pm 0.8^{\mathrm{a}}$ & $10.3 \pm 0.9^{\mathrm{ab}}$ \\
\hline PUFA & $38.9 \pm 3.0^{\mathrm{a}}$ & $39.3 \pm 2.2^{\mathrm{a}}$ & $40.8 \pm 3.3^{\mathrm{a}}$ & $34.5 \pm 1.8^{\mathrm{a}}$ & $36.6 \pm 0.7^{\mathrm{a}}$ \\
\hline$n-3$ & $30.4 \pm 3.2^{\mathrm{a}}$ & $28.7 \pm 1.3^{\mathrm{a}}$ & $28.5 \pm 1.2^{\mathrm{a}}$ & $26.9 \pm 2.1^{\mathrm{a}}$ & $27.5 \pm 0.9^{\mathrm{a}}$ \\
\hline$n-6$ & $7.5 \pm 1.0^{\mathrm{ab}}$ & $10 . \pm 1.1^{\mathrm{ab}}$ & $11.6 \pm 2.9^{\mathrm{a}}$ & $6.8 \pm 0.6^{\mathrm{bc}}$ & $8.3 \pm 0.5^{\mathrm{ab}}$ \\
\hline$n-3 / n-6$ & $4.8^{\mathrm{a}}$ & $3.0^{\mathrm{ab}}$ & $2.9^{\mathrm{ab}}$ & $4.2^{\mathrm{ab}}$ & $3.4^{\mathrm{ab}}$ \\
\hline $\mathrm{EPA}+\mathrm{DHA}$ & $24.7 \pm 2.8^{\mathrm{a}}$ & $24.6 \pm 1.3^{\mathrm{a}}$ & $22.6 \pm 1.9^{\mathrm{a}}$ & $22.4 \pm 2.1^{\mathrm{a}}$ & $23.1 \pm 1.0^{\mathrm{a}}$ \\
\hline $\mathrm{EPA}+\mathrm{DHA}^{*}$ & 443 & 270 & 316 & 246 & 600 \\
\hline PI & 234 & 238 & 246 & 211 & 220 \\
\hline $\mathrm{HH}$ & 1.72 & 2.0 & 1.96 & 1.66 & 1.82 \\
\hline
\end{tabular}

PI: peroxidisability index $=(0.025 \times$ monoenes $)+(1 \times$ dienes $)+(2 \times$ trienes $)+(4 \times$ tetraenes $)+(6 \times$ pentaenes $)+(8 \times$ hexaenes $)$. HH: hypocholesterolaemic/hypercholesterolaemic ratio $=\left(\mathrm{C}_{18: 1 \mathrm{n}-9}+\mathrm{C}_{18: 2 \mathrm{n}-6}+\mathrm{C}_{20: 4 \mathrm{n}-6}+\mathrm{C}_{18: 3 \mathrm{n}-3}+\mathrm{C}_{20: 5 \mathrm{n}-3}+\mathrm{C}_{22: 5 \mathrm{n}-3}+\mathrm{C}_{22: 6 \mathrm{n}-3}\right) /\left(\mathrm{C}_{14: 0}+\mathrm{C}_{16: 0}\right)$. EPA + DHA ${ }^{*}: \mathrm{mg}_{1} / 100 \mathrm{~g}$ de poisson.

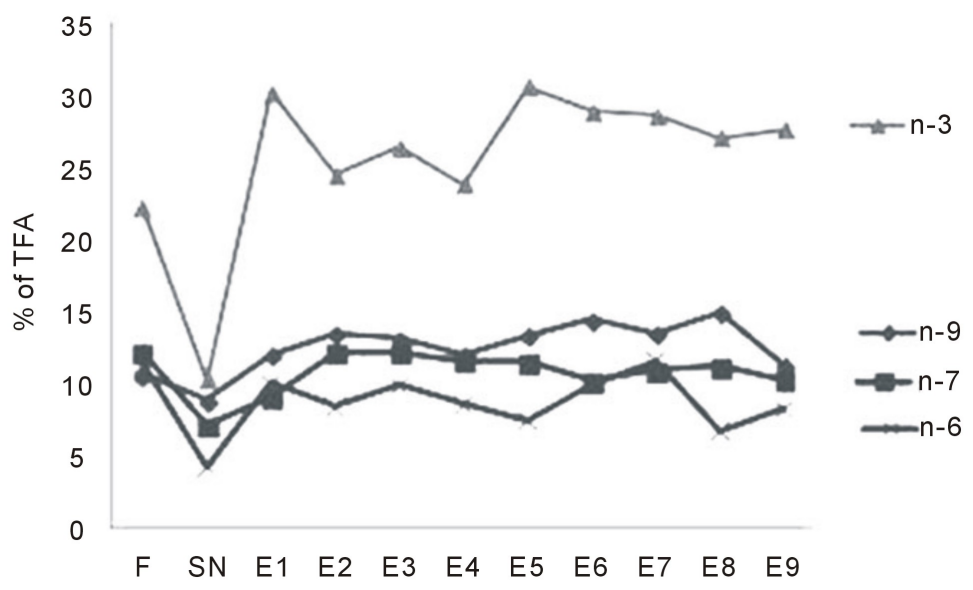

Figure 2. Percentages of n-3, n-6, n-7, and n-9 fatty acids under different experimental conditions. 
in $\mathrm{mg} / 100 \mathrm{~g}$ of fish. These levels varied depending on the experimental conditions. The levels were higher than $300 \mathrm{mg} / 100 \mathrm{~g}$ except with natural drying (SN), E6, and E8 where the values were lower than $300 \mathrm{mg} / 100 \mathrm{~g}$.

The peroxidisability index (PI) represents the relationship between the fatty acid composition of tissue and its susceptibility to oxidation. This index showed high values of 246 and 238 in E1, E7, and E6, respectively, and a value of 187 for E4. For the hypocholesterolemic/hypercholesterolemic ratio, the values were 2 and 1.96 in E6 and E7, respectively.

\section{Discussion}

The drying of fish is performed to reduce some of the so-called "free" water that is a vector of various infections and is involved in the degradation reactions. Drying helps promote preservation. During natural drying, the fish is exposed to air and natural light. Drying in the sun can be performed for 3 to 10 days, but drying times of 1 to 3 days are more common [15].

The protein content of fresh A. boyeri was 18.3\%. With natural drying, the surface of the fish dries faster and hardens, locking moisture inside, which slows down the drying process and promotes the degradation of proteins [16]. In this study, we obtained a protein content of $14.2 \mathrm{~g} / 100 \mathrm{~g}$. Protein destruction is accelerated when the products are subjected to high temperatures for an extended period of time [16]. The total fatty acid (TFA) content of fish affects the biochemical characteristics postmortem. In this study, the TFA content of fresh fish was $4.9 \mathrm{~g} / 100 \mathrm{~g}$; it was $0.5 \mathrm{~g} / 100 \mathrm{~g}$ with sun-dried fish. When drying conditions change (E1 to E9), the contents of total fatty acids vary from $1.1 \mathrm{~g} / 100 \mathrm{~g}$ to $2.8 \mathrm{~g} / 100 \mathrm{~g}$.

The exposure of A. boyeri for extended periods to solar drying led to the degradation of lipids. The formation of lipid oxidation by-products reduces the nutritional quality and increases health risks [17] because they are associated with aging, altered membranes, heart disease, and cancer [18]. Photo-oxidation explains the low trans fat content of fish exposed to the air [19].

Taking into account productivity, one might think that drying with warm air would take less time than natural drying. In E2, E3, E4, and E9, the TFA content increased in parallel with the temperature. However, a high temperature of $70^{\circ} \mathrm{C}$ (E1) can alter the final TFA content, which was $1.8 \mathrm{~g} / 100 \mathrm{~g}$. It seems that the $\mathrm{E} 2$ conditions would be ideal for the preservation of AGT in A. boyeri.

In fresh A. boyeri, palmitic acid was the major component of the SFAs, with a relative percentage of $57.5 \%$ (of SFAs), followed by stearic acid with a relative percentage of $29.3 \%$ (of SFAs). The profile of fatty acids in $A$. boyeri was distinguished by the presence of myristic acid $\left(\mathrm{C}_{14: 0}\right)$, with a relative percentage of $9 \%$ (of SFA). During natural drying, the respective percentages of these fatty acids were $51.4 \%, 42.2 \%$, and $2.9 \%$ (of SFA). These three fatty acids cannot be considered as a whole because they differ in their structure, metabolism, cellular functions, and even their deleterious effects in case of excess [20]. Myristic acid represents a small proportion of the TFAs in the animal body (between $0.5 \%$ and $2 \%$ of TFA) [21].

The three main MUFAs in fresh $A$. boyeri were palmitoleic acid $\left(\mathrm{C}_{16: 1 \mathrm{n}-7}\right)$, oleic acid $\left(\mathrm{C}_{18: 1 \mathrm{n}-9}\right)$, and vaccenic acid $\left(\mathrm{C}_{18: 1 \mathrm{n}-7}\right)$, with relative percentages of $24.6 \%, 44 \%$, and $28.2 \%$ (of MUFA). These three fatty acids were identified as major fatty acids in natural drying, with relative percentages of $13 \%, 55.6 \%$, and $31.2 \%$ (of MUFA), respectively. Oleic acid is characteristic of fish tissue [22] and is actively synthesized by the cells [23]. Under the action of ACAT (acylCoA-cholesterol acyltransferase), oleic acid binds to cholesterol [23]. Cholesterol esters thus formed represent the form of the transport of cholesterol in lipoproteins [22]. Under experimental drying, the highest percentage of oleic acid was observed with E6 and E8, with 57.7\% and 55.4\% (MUFA), respectively.

A. boyeri, in addition to being a source of protein, contains large amounts of PUFAs. In fresh fish, the percentage was $34.7 \%$ (of TFA), and between $33.2 \%$ and $41.4 \%$ (TFA) with experimental drying. The n-6 PUFA content was $11 \%$ (of TFA) in fresh fish. This category is represented by linoleic acid (LA, C18:2n-6), acid which has a percentage of $5.1 \%$ and by arachidonic acid (ARA, $\mathrm{C}_{20: 4 n-6)}$ ) with a respective percentage of $5 \%$ (TFA). As shown in Table 5, under different experimental conditions, ARA was the main n-6 PUFA except with E5. The percentages of n-3 PUFAs were higher than those of n- 6 PUFAs, as shown by the n-3/n- 6 ratios in Table 5 . The highest ratios of 4.8 and 4.2 were observed with E5 and E8, respectively. This ratio is useful for comparing the nutritional value of fish oils [24]. Fish and fishery products rich in n-3 fatty acids and low in n-6 fatty acids are considered good for human health [25]. An increase in the n-3/n-6 ratio is essential to help the body use n-3 fatty acids. A low ratio indicates that the enzymes that convert fatty acids to their active forms are likely to be used by 
n-6 PUFAs [26]. The percentage of EPA + DHA is responsible for variations in the n-3/n-6 ratios [26].

Research has shown that there are significant health benefits of a diet rich in EPA and DHA. DHA and ARA are important in neonatal health [27] [28], in particular in eye and brain development. A number of countries including Canada and the United Kingdom, and organizations such as the World Health Organization (WHO) and North Atlantic Treaty Organization have advocated dietary recommendations for n-3 PUFAs. These recommendations are 0.300 to $0.500 \mathrm{mg} /$ day EPA + DHA [29]. Based on the results in Table 5, these recommendations can easily be met by consuming $100 \mathrm{~g}$ fresh A. boyeri (i.e., $680 \mathrm{mg}$ EPA + DHA), or $200 \mathrm{~g}$ of dried fish with E6 (540 mg EPA + DHA), E7 (632 mg EPA + DHA) or E8 (492 mg EPA + DHA).

The PI index [30] shows the relationship between the unsaturated fatty acid composition of tissue and its susceptibility to oxidation. It provides information on the quality of the product that when the value of PI is high, polyunsaturated fatty acids are susceptible to be oxidized. The higher the PI value, the higher fat fish is likely to be oxidized. Factors that influence lipid oxidation are either intrinsic, such as the fatty acid composition of lipids (number and position of unsaturation), the presence of pro-oxidants (heme, metal ions, and enzymes) or natural antioxidants (tocopherols and carotenoids), and external factors such as temperature, light, oxygen, water activity, and the conditions of storage and processing [31]. The PI of fresh A. boyeri tissue was 176; the PI of sundried A. boyeri was 83. Although the conditions E1, E5, E6, and E7 had the highest values on the PI index, they also showed a greater sensitivity to oxidation than other conditions. The values that were closest to that of fresh fish were 187 (E2) and 195 (E4). The values that are closest to fresh fish are 187 and 195 and they correspond to drying conditions E4 and E2.

The relationship between hypocholesterolaemic and hypercholesterolaemic fatty acids (HH) [32] provides information on the effects of specific fatty acids on cholesterol metabolism. The highest ratios that are most beneficial were found with E6 (2), E7 (1.96), E1 (1.82), and E9 (1.82).

\section{Acknowledgements}

The authors thank Mrs. Hedia Chaabane who contributed to the achievement of this work.

\section{References}

[1] Robb, D.H.F., Kestin, S.C., Warriss, P.D. and Nute, G.R. (2002) Muscle Lipid Content Determines the Eating Quality of Smoked and Cooked Atlantic Salmon (Salmo Salar). Aquaculture, 205, 345-358. http://dx.doi.org/10.1016/S0044-8486(01)00710-4

[2] Dumay, J. (2006) Extraction de lipides en voie aqueuse par bio-réacteur enzymatique combiné à l'ultracentrifugation: Application à la valorisation de co-produits de poisson (Sardina pilchardus). Ph.D. Dissertation, Ecole Polytechnique de l’Université de Nantes, 283. http://archimer.ifremer.fr/doc/2006/these-1556.pdf

[3] Harper, C.R. and Jacobson, T.A. (1987) The Role of Omega-3 Fatty Acids in the Prevention of Coronary Heart Disease. Archives of Internal Medicine, 161, 2185-2192. http://dx.doi.org/10.1001/archinte.161.18.2185

[4] Piclet, G. (1987) Le poisson aliment. Composition-Intérêt nutritionnel. Cahiers de la Nutrition et de la Diététique, 4, 317-336.

[5] Médale, F., Lefèvre, F. and Corraze, G. (2003) Qualité nutritionnelle et diététique des poissons. Cahiers de Nutrition et de Diététique, 38, 37-44.

[6] Okland, H.M.W., Stoknes, I.S., Remme, J.F., Kjerstad, M. and Synnes, M. (2005) Proximate Composition, Fatty Acid and Lipid Class Composition of the Muscle from Deep Sea Teleosts and Elasmobranchs. Comparative Biochemistry and Physiology_Part B, 140, 437-443. http://dx.doi.org/10.1016/j.cbpc.2004.11.008

[7] Grégoire, F., Dionne, H. and Lévesque, C. (1994) Contenu en gras chez le maquereau bleu (Scomber scombrus L.) en 1991 et 1992. Rapport canadien à l'Industrie sur les sciences halieutiques et aquatiques, 220, 82.

[8] FAO (2008) Assurer l'approvisionnement régional des produits aquatiques: Perspectives suite aux études de cas de la FAO dans l'évaluation des pertes post-capture-Comité des pêches continentales et de l'aquaculture pour Afrique, Quinzième session. FAO, Lusaka.

[9] Choubert, G. (2010) Procédés de conservation/transformation et qualité sensorielle du poisson. $13^{\text {ème }}$ Journées Sciences des Muscles et Technologies des Viandes, Clermont-Ferrand, 19-20 Octobre 2010, 91-98.

[10] Knockaert, C. (1990) Le fumage du poisson. http://archimer.ifremer.fr/doc/00004/11490/

[11] Bouriga, N., Ben Alaya, H., Selmi, S., Azouz, S., Faure, E. and Trabelsi, M. (2009) Effets de deux procédés de séchage sur la qualité lipidique des athérines de l'île de Djerba (Tunisie). Société des Sciences Naturelles de Tunisie, 36, 44-51. 
[12] Folch, J., Lees, M. and Sloane-Stanley, G.A. (1957) A Simple Method for the Isolation and Purification of Total Lipids from Animal Tissues. Journal of Biological Chemistry, 226, 497-509.

[13] Cecchi, G., Basini, S. and Castano, C. (1985) Méthanolyse rapide des huiles en solvant. Revue française des corps gras, 4, 163-164.

[14] Lowry, O.H., Rosebrough, N.J., Farr, A.L. and Randall, R.J. (1951) Protein Measurement with the Folin Phenol Reagent. Journal of Biological Chemistry, 193, 265-275.

[15] FAO/UN (1992) Fish and Fuel. Food and Forests. Perspectives on Post-Harves Losses in Uganda. Fishin Project: P O Box 521. FAO/UN, Kampala.

[16] Ariyawansa, S. (2000) The Evaluation of Functional Properties of Fish Meal. United Nations University, Fisheries Training Programme, Project Final, Sri Lanka, 1-25.

[17] Frankel, E.N. (1996) Antioxidants in Lipid Foods and Their Impact on Food Quality. Food Chemistry, 57, 51-55. http://dx.doi.org/10.1016/0308-8146(96)00067-2

[18] Suja, K.P., Abraham, J.T., Thamizh, S.N., Jayalekshmy, A. and Arumughan, C. (2004) Antioxidant Efficacy of Sesame Cake Extract in Vegetable Oil Protection. Food Chemistry, 84, 393-400. http://dx.doi.org/10.1016/S0308-8146(03)00248-6

[19] Ashton, I.P. (2002) Understanding Lipid Oxidation in Fish. In: Bremmer, H.A., Ed., Safety and Quality Issues in Fish Processing, Woodhead Publishing Limited, Cambridge, 254-285. http://dx.doi.org/10.1533/9781855736788.2.254

[20] Agence Française de Sécurité Sanitaire des Aliments (2010) Avis de l’Agence Française de Sécurité Sanitaire des Aliments. Actualisation des apports nutritionnels conseillés pour les acides gras, Saisie 2006-SA-0359, 1-10.

[21] Rioux, V. and Legrand, P. (2001) Métabolisme et fonctions de l'acide myristique. Oléagineux, Corps Gras Lipides, 8, 161-166.

[22] Steffens, W. (1997) Effects of Variation in Essential Fatty Acids in Fish Feeds on Nutritive Value of Freshwater Fish for Humans. Aquaculture, 151, 97-119. http://dx.doi.org/10.1016/S0044-8486(96)01493-7

[23] Legrand, P. (2007) Les acides gras: Structures, fonctions Apports nutritionnels conseillés. Cahiers de Nutrition Diététique, 42, 7-12.

[24] Pigott, G.M. and Tucker, B.W. (1990) Seafood: Effects of Technology on Nutrition. Marcel Dekker, Inc., New York.

[25] Sargent, J.R. (1997) Fish Oils and Human Diet. British Journal of Nutrtion, 78, 5-13. http://dx.doi.org/10.1079/BJN19970131

[26] Hossain, M.A. (2011) Fish as Source of n-3 Polyunsaturated Fatty Acids (PUFAs), Which One Is Better-Farmed or Wild? Advance Journal of Food Science and Technology, 3, 455-466.

[27] Horrocks, L.A. and Yeo, Y.K. (1999) Health Benefits of Docosahexaenoic Acid (DHA). Pharmacological Research, 40, 211-225. http://dx.doi.org/10.1006/phrs.1999.0495

[28] Innis, M.S. (2000) The Role Dietary n-6 and n-3 Fatty Acids in the Developing Brain. Developmental Neuroscience, 22, 474-480. http://dx.doi.org/10.1159/000017478

[29] Kris-Etherton, P.M., Harris, W.S. and Appel, L.J. (2002) Fish Consumption, Fish Oil, Omega-3 Fatty Acid and Cardiovascular Disease. Circulation, 106, 2747-2757. http://dx.doi.org/10.1161/01.CIR.0000038493.65177.94

[30] Erickson, M.C. (1992) Variation of Lipid and Tocopherol Composition in Three Strains of Channel Catfish (Ictalurus punctatus). Journal of the Science of Food and Agriculture, 59, 529-536. http://dx.doi.org/10.1002/jsfa.2740590416

[31] Hsieh, R.J. and Kinsella, J.E. (1989) Lipoxygenase Generation of Specific Volatile Flavor Carbonyl Compounds in Fish Tissues. Journal of Agricultural and Food Chemistry, 37, 279-286. http://dx.doi.org/10.1021/jf00086a001

[32] Santos-Silva, J., Bessa, R.J.B. and Santos-Silva, F. (2002) Effect of Genotype, Feeding System and Slaughter Weight on the Quality of Light Lambs. II. Fatty Acid Composition of Mea. Livestock Production Science, 77, 187-194. http://dx.doi.org/10.1016/S0301-6226(02)00059-3 
Scientific Research Publishing (SCIRP) is one of the largest Open Access journal publishers. It is currently publishing more than 200 open access, online, peer-reviewed journals covering a wide range of academic disciplines. SCIRP serves the worldwide academic communities and contributes to the progress and application of science with its publication.

Other selected journals from SCIRP are listed as below. Submit your manuscript to us via either submit@scirp.org or Online Submission Portal.
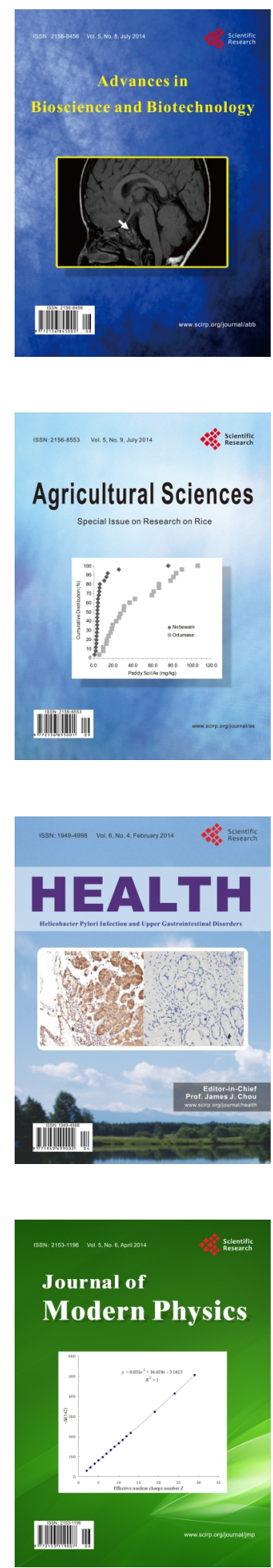
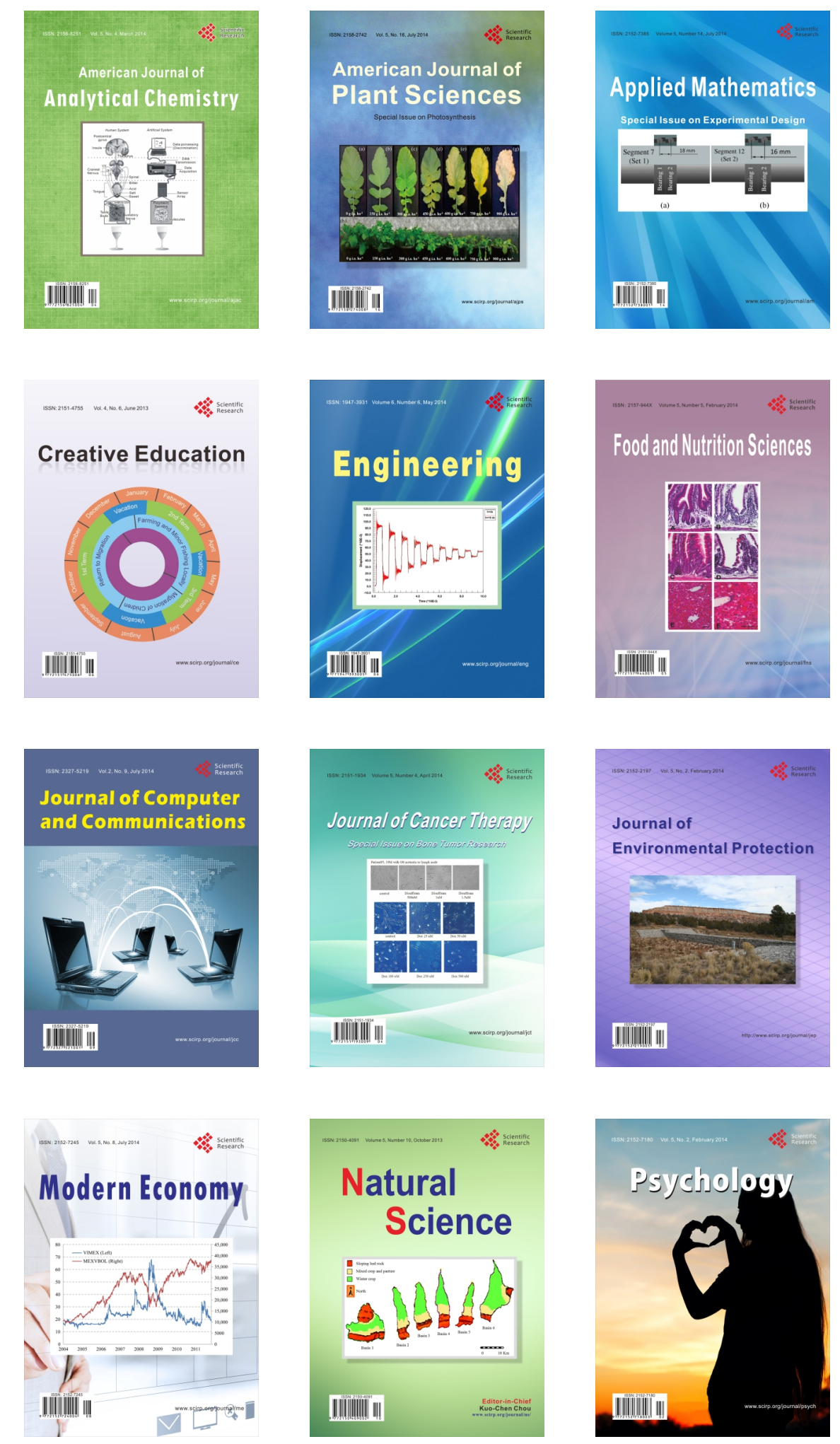